\title{
QUANTITATIVE MORPHOLOGICAL CHANGES OF THE CHOROIDAL VASCULATURE IN NORMAL AGED AND DISEASED HUMAN DONOR EYES
}

\author{
Swathi Kanduri \\ The University of Auckland Department of Ophthalmology Faculty of Medical and Health Sciences \\ Grafton, Auckland New Zealand
}

\begin{abstract}
Introduction: To analyze whether the choriocapillaries undergo degenerative changes with age and disease. Corrosion casts of choroidal vessels in human donors were investigated by scanning electron microscopy (SEM) to understand the vasculature changes with age.

Method: The patterns of blood vessels, vortex veins, ciliary arteries placements, capillaries placement from centre to periphery in different quadrants and regions of choroid were evaluated in twenty donor normal tissues aged from 20-90 years. Nine donors had diabetic donor tissues were evaluated in which different quadrants with laser marks and loss of blood vessels were quantified along with, six donors with signs of agerelated macular degeneration were evaluated using a light stereoscopic dissection microscope. Six human donors were investigated by SEM of which two tissues were normal, two had diabetic retinopathy and two presented with age related macular degeneration changes.

Result: The quantitative evaluation revealed that in normal to diseased there is a decrease of the blood vessel density in comparison to the total area of the choroid. There was also slight decrease from to centre to periphery in the \% area of the blood vessel placement. Morphological identification noted was a greater number of vortex veins were noted in inferior end than the superior end. There was prominent loss of blood vessel bed was noted beneath the lasered areas in diabetic retinopathy tissues.

Conclusion: Loss of \% area of blood vessel density was directly correlating with the progression of age and in diabetic retinopathy diseased tissues we noted prominent loss of blood vessels underneath the laser treated areas. In age related macular degeneration, loss of blood vessels was prominent in different quadrants with age too.
\end{abstract}

Keywords: Choriodal Vasculature, Morphological changes in choroid, vascular changes in AMD and DR Cite This Article: KANDURI, Swathi. Quantitative Morphological Changes of the Choroidal Vasculature in Normal Aged and Diseased Human Donor eyes. International Journal of Retina, [S.I.], v. 4, n. 1, p. 58, feb. 2021. ISSN 2614-8536. Available at: <https://www.ijretina.com/index.php/ijretina/article/view/135>. Date accessed: 22 feb. 2021. doi: https://doi.org/10.35479/ijretina.2021.vol004.iss001.135.

${ }^{*}$ Correspondence to:

Swathi Kanduri,

University of Auckland

Auckland, New Zealand

swathichary@gmail.com

\section{INTRODUCTION}

The vascular diseases of retina are diagnosed by clinicians based on the appearance of the retinal blood vessel patterns and the articulator of artery to vein ratio in the posterior segment of the eye. ${ }^{1}$ The signs of late stages of the retinal diseases include blood vessel changes such as leakage or growth of new blood vessels, these signs are important for the treatment of the ocular vascular diseases. ${ }^{2}$ Recent literature shows evidence of changes in the blood flow and choroidal vasculature in the early stages of the retinal diseases such as age-related macular degeneration (AMD). These changes in the patients with AMD attributes to categorise them as vascular pathology. ${ }^{3}$ There is an association noted between some systemic diseases and ocular vasculature manifestation. For an example, such as diabetes leading to diabetic retinopathy (DR), hypertension, arteriosclerosis and cardiovascular diseases. Dilated fundus examination of the eyes 
shows vascular changes in correspondence to these systemic diseases. ${ }^{4,5}$ Studies show that currently there are no treatments available for treating the early stages of the diseases AMD and DR. Choroidal vasculature changes are noted in the early stages of these diseases. Understanding and treating the choroidal vasculature changes may help in early treatment of these diseases. ${ }^{6}$ The evaluation of the retinal blood vessel pattern is important in understanding these diseases as progression occurs rapidly in them. The current ideal clinical recommendation is to follow up these patients with ocular vascular problems, every six months. 7,8,9 Previous studies show effective ways for identifying these diseases AMD and DR in early stages by using computer aided retinal blood vessel segmentation programmes. $^{10,11 \quad \text { Furthermore, automated }}$ segmentation models have been developed for evaluating blood vessel changes in these diseases. 12,13 These computerised algorithms help in identification of loss of blood vessel by tracking method. Edge-detection, pattern recognition models are also useful in studying the retinal blood vessels. $13,14,15,16,17$ In this study, we hypothesized that these age-related retinal degeneration diseases are associated with vascular changes in the choroid that can be detected in the early stages using pattern recognition of segmentation model. The human post-mortem tissues are ideal for studying the choroidal vasculature pattern as they are replica of human eyes and ideal for the development of new algorithm.

\section{METHOD}

\section{The human donor eyes usage in research:}

The donor tissues were received by the New Zealand National Eye Bank. The Northern B Health and Disability Ethics Committee has approved usage of human donor tissues for research in the University of Auckland, New Zealand. All the study procedures involving human donor tissues were conducted in accordance with the Declaration of
Helsinki. Thirty-six donor tissues aged 20-90 years were used in this study. Human donor tissues were processed for optical coherence tomography (OCT) imaging analysis. To study the vasculature changes seen through the OCT analysis, we have developed an algorithm for studying the choroidal blood vessel patterns using vessel segmentation model. The algorithm used in this study is "An Image J program" (National Institute of Health, Maryland, USA). It is a built-in algorithm based on image processing technique. The algorithm helps in identification of the blood vessels and to define the blood vessel area by detecting the difference in contrast between the blood vessel wall and the choroid pillars. Quantification of blood vessel density was done using the computerised algorithm in the choroids of the normal young donors and in aged tissues in comparison with diseased (AMD and DR donor eyes). In this study, we have also included the ultrastructural corrosion cast analysis to visualize the choroid blood vessel patterns and to confirm whether blood vessel density changes were associated with a remodelling in the in these diseases. ${ }^{18}$

\section{Tissue imaging and analysis}

Micron IV OCT equipment (Phoenix Research Laboratories, USA) was used to conduct seven field fundus imaging and optical coherence tomography (OCT) techniques. Imaging of the posterior segment of the eye was performed using a purpose-build holder. Poly gel (containing $3 \mathrm{mg} / \mathrm{g}$ Carbomer, Alcon Laboratories, Pty Ltd., New South Wales, Australia) was filled in the posterior segments of the donor eye before imaging to reduce glare during imaging of the eyecup. The donor retinal images obtained were graded based on standard clinical classification of AMD and DR pathology grading. Based on fundus photographs and OCT images obtained, twenty-one donor eyes (20-90 years old) were confirmed having no ocular pathology and was categorised as normal. In nine 
donor tissues (aged 60-90 years) haemorrhages and laser marks were noted through OCT and fundus imaging. In addition to donor information sheet stating the donors had systemic diabetes. Thus, confirming these donor tissues with diabetic retinopathy (DR). Confirmed presence of drusen and haemorrhages with fibrosis through fundus imaging and OCT in six donor tissues (aged 60-90 years) these are categorised as age related macular degeneration (AMD). For few of these donor eyes the donor information sheet, had a mention of the AMD condition.

\section{Dissection procedure performed to separate} retina and choroidal layers: After cornea's being used by the eye bank for transplantation process the posterior segments were received for the research. The freshly received posterior segments were dissected to obtain separate choroid layers. Dissection was done using sterile Westcott Scissors (Storz Ophthalmics E3320, country). The vitreous, clear jelly like structure was removed first, with no or minimal damage to underlying layers of the globe. There was minimal vitreous attached above the optic disc to avoid retinal detachment in the globe. Holding with a tight grip on the sclera at one end with forceps the scissors were passed in the globe to separate the layers retina and choroid. The retina and choroid were separated underneath the RPE layer. Gentle running of the scissors allowed separation of the two layers with no damage but there was still some pigmentation attached at one of the edges either superior or inferior. The scalpel blade was used to dissect out the remaining (nonretina) tissue. Under Leica dissecting microscope 100x objective lens we have visualized separate layers of sclera, retina and choroid and evaluate the damage to the tissue (if any). Bright field microscopy (Leica Bright field microscope DM-RA, Danaher Corporation, Germany) was used to read the obtained choroids. It was blackish brown pigmentated layer and abundant different sized blood vessels were seen within a thick matrix. The single sectorial colour images were captured using a digital colour camera (Leica Microsystems CMS $\mathrm{GmbH}$. Leica application suite version 2.5.0 R1) under 100-x objective lens. The single images obtained by the digital camera were converted into a montage full image of size $565 \times 580$ pixels using Image J software (National Institute of Health, Maryland, USA). These images were then used in quantification using computerised algorithms like segmentation model and edge detection tools.

\section{Image - J image processing technique - The Segmentation method}

To quantify the blood vessel layer - the choroid a convolution algorithm called segmentation method was developed. All the dissected and extracted choroid images were converted into a grey scale image, with loss of Red Green Blue (RGB) pixels in the images as described in the previous studies. 19,20,21 The normalisation of these images was done by equal subtraction of the pixel values from the original background image pixel values. The 3x 3-pixel matrix was applied on these images to smoothen and reduce the noise from the surrounding structures of the blood vessels, such as tissue borders and matrix. The average intensity of the pixels was calculated and was used in the $3 \times 3$ pixel matrix to smoothen the blurred areas. To estimate the background pixel values in these images, a large arithmetic mean kernel was used..$^{22}$ The neighbourhood pixels of first the radius were measured and averaged to the validated the obtained background pixel values. ${ }^{23}$ In Image J software, the mean filter calculation was done using the following formula: Application of the kernel was done at the centre of image and the same kernel was superimposed across the image until periphery to standardise each pixel value in the total image. Line detection filters were applied to enhance the blood vessel contrast of each image..$^{24,25,26,27}$ For final validation of the segmentation method obtained pixel values the computation of the mean 
and maximum pixel values were combined. 28,29 Setting called dilated was applied in Image J to read these images. This application helps in analysing and understating the unnecessary expand of the area in the images and to avoid the gaps between the images. The optic disc is the centre of the fundus image. The blood vessels in the retina branches out from this cup. In choroid layer, the disc area appears hallow as gap area. For maintaining the uniformity in reading the choroid images the hallow area obtained from disc was treated with the dilated setting. This treatment avoided confusion in application of the segmentation method analysis. Closing operator is another application in the Image $J$ used to preserve background areas with uniformity. The specified pixel values obtained from RGB images were matched in the grey scale values using the, impixel operator. ${ }^{30,31,32}$ To maintain the contrast through out in these images the histogram equalization tool was used in the Image J software. A combination of binary image thresholding procedure was used to detect the blood vessels (pixel value 1) and background space as (pixel value 0). The threshold process helped in identification of the unwanted noise in the images (as false positive pixel values). Further treatment of the images was done using convolution method to avoid these false positives and final images were obtained with the desired results. ${ }^{33}$ Furthermore, other filters were applied in Image $J$ to refine and obtain edge detection in these binary images. ${ }^{34,35}$

\section{The choroid vasculature imaging by scanning electron microscopy (SEM)}

For conducting SEM imaging, we have used two human donor tissues of each normal, DR and AMD. The choroids were dissected and extracted out carefully from the posterior segment for analysis as stated above. The dissected and extracted choroid tissues were incubated for $12 \mathrm{hrs}$ at $4^{\circ} \mathrm{C}$ placing in $2.5 \%$ glutaraldehyde $+0.1 \mathrm{M}$ phosphate buffer (PB).
Then, for every 30 minutes, the tissues were washed in the buffer $0.1 \mathrm{M}$ PB thrice and were incubated overnight at $4{ }^{\circ} \mathrm{C}$ again in $0.1 \mathrm{M} \mathrm{PB}$ buffer. Followed by the overnight incubation, $1 \mathrm{~mm}$ sized choroid tissues were post fixed in $1 \%$ osmium tetroxide in $0.1 \mathrm{M} \mathrm{PB}$ for $1 \mathrm{hr}$ at room temperature. Following the incubation, at room temperature all the tissues underwent dehydration process through changing in ethanol solution for every 10-minutes (ethanol solutions used were $50 \%, 70 \%, 90 \%$ and $100 \%$ of concentrations). For completion of the dehydration process, all these tissues had extra wash steps in $100 \%$ ethanol solution for every 20 minutes twice. Polaron (model E3000, company) machine was used for drying out all the tissues. After completion of drying process, all these tissues were mounted onto SEM stubs with sclera side of the tissue facing down. Before imaging, DSR1 Desk Sputter Coater (Nanostructured Coatings Co., Iran) machine was used to gold coat these all specimens and threedimensional scanning electron microscopy (Hitachi TM3030Plus Tabletop Microscope, (Hitachi High Technologies Corporation, Tokyo, Japan) was used to view and read the choroid structures.

\section{Data Analysis}

Statistical analysis was conducted using One-way ANOVA followed by Bonferroni post hoc test in Graph Pad Prism 7 (Graph Pad Software, Inc.CA). All the data were presented as mean \pm standard error of the mean. Quadrants of the choroid were defined as superior, superior temporal, superior nasal, inferior, inferior nasal and inferior temporal, nasal and temporal regions. Comparison between groups was statistical significantly when $p<0.05$.

\section{RESULT}

In this study, we have reconstructed thirty-six images of flat mounted choroids to evaluate the blood vessel areas using the convolution algorithm. Twenty-one from control eyes and fifteen from $A M D$ and $D R$ diseased eyes were used. To maintain 
the consistency throughout image analysis, all the images were processed with same settings in all steps.

Figure1, defines in detail the steps taken to process the choroidal tissues and to obtain their images to apply the convolution blood vessel segmentation algorithm. Donor fundus image is shown in the Figure 1a which includes both retina and choroid layers. Figure $1 b$ is the separated retina from choroid, showing vasculature of the retina. In this study we will not be using these images for the analysis. Figure $1 \mathrm{c}$ represents the choroidal vasculature in detail from centre to periphery. Figure $1 \mathrm{~d}$ is the enhanced grey scale conversed image of the choroid with all the quadrant details as seen in Figure $1 \mathrm{c}$. Figure $1 \mathrm{e}$ is the representative image of the choroid after the application of convolution mathematical algorithm. The blood vessels patterns, borders and density are unique and well represented. Figure $1 \mathrm{f}$ is the quantification of the image in seven different quadrants covering all the total area of the choroid. The mathematical algorithm helped to demarcate the patterns of blood vessels in normal and diseased tissues. In diseased DR donor eyes, fundus images show signs of haemorrhages, pigmentation and laser treatment (Figure 1-2a, b) which are well replicated in the Figure $1-2 c$ in the grey scale image. Figure 1$2 \mathrm{~d}$ is the algorithm treated image. The segmentation model enhances the areas occupied by the blood vessels and shows dark spaces in between the vessels which is the representation of loss of tissue due to the laser treatment. Figure 1$3 a, b$ are the fundus images with signs of AMD donor eyes such as drusen. The grey scale image of AMD donor Figure 1-3c and the algorithm applied image Figure 1-3d reveals the decreased density of the blood vessels and disturbed patterns in AMD choroid.

Seven quadrant quantification was performed to analyse the total area of the choroidal blood vessel density. In normal tissues, across the groups and within each quadrant F1-F7 there was no statistically significant decrease in the density of blood vessels was noted. However, in the inferior nasal quadrant (F7), statistically significant decrease in the blood vessel density was noted of all quadrants (Figure 1-4-7). statistical significance was noted in analysis of diseased tissues per quadrant in comparison with age-matched controls in each quadrant. In addition, statistically significant decrease was noted in the blood vessel density in comparison between central quadrants average value from F1.F2.F3 to the peripheral F4, F5, F6, F7 average; which was $16.9 \%$.

Total choroid blood vessel density area analysis ( $n=3$ per group) showed progressive decline in blood vessel density in the normal choroid in comparison with age matched normal. The normal donor choroids 20-30 years of age revealed mean \pm SEM $37.5 \% \pm 0.8$ of area was occupied by the blood vessels, in comparison with the normal donors aged $81-90$ years have $30.3 \% \pm 1.59$.

All the DR donor tissues ( $n=3$ per age group), analysis of total blood vessel area showed (60-70 years) mean \pm SEM $19.8 \pm 2.6$, wherein, there was a statistically significant decrease noted in total blood vessel area with age 71-80 years $16.9 \pm 2.6$ and 81-90 years showed $14.4 \pm 2.3$. DR choroids had a $77 \%$ decrease in blood vessels when compared with age-matched DR controls. Furthermore, we have noted that $60-90$ years of age DR tissues showed $6.52 \%$ further drop in blood vessels and all of them presented with laser marks in different quadrants.

Total area of blood vessels in age matched AMD choroids tissues ( $\mathrm{n}=3$ per age group) noted was 71 80 years had $22.7 \pm 1.7$. There was no statistically significant decrease in blood vessel density was noted $80-90$ years age of donors $21.2 \pm 1.3$. Total of $5.61 \%$ decrease in blood vessels density was noted in diseased AMD tissues.

3D - SEM imaging analysis, Figure 1.6 reveals that donor tissues are normal. The anatomical view of 
the choroid with presence of choriocapillaris at the centre and different sized blood vessels (small, medium and large sized blood vessels) at periphery. The matrix is compact configuration within the tissue. Images $1-6(1 \mathrm{~mm}$ to $100 \mu \mathrm{m} ; \mathrm{A}-\mathrm{G})$ shows larger to smaller tissue areas in normal tissues. Details of the choroidal vascular structures are revealed in higher magnifications $(100 \mu \mathrm{m})$ such as larger blood vessels, branching into small capillary meshwork in the choroid.

SEM of AMD diseased tissues, Figure 1.7 revealed soft drusens and loss of configuration of the choroidal tissues. All the images from lower to higher magnification (1.7 A-C, D-F) confirmed drusen are small, confluent ball structures (30-
$100 \mu \mathrm{m})$. The loss of structural integrity and disturbed patterns of blood vessels were noted in these tissues.

SEM of DR donor tissues, Figure 1.8 shows spares in the tissues especially underneath the lasered treated areas confirming the loss of blood vessel patterns. Loss of composition of the tissues was confirmed from the images (1.8 A-3C, 3D-3F). The spaces and holes within the matrix of tissues was confirmed in the grey scale images of the DR donor eyes treated with laser. The images were obtained from different magnification ranges of $1 \mathrm{~mm}$ to $300 \mu \mathrm{m}$ and all confirmed loss of patterns of blood vessels.
Normal central Fundus

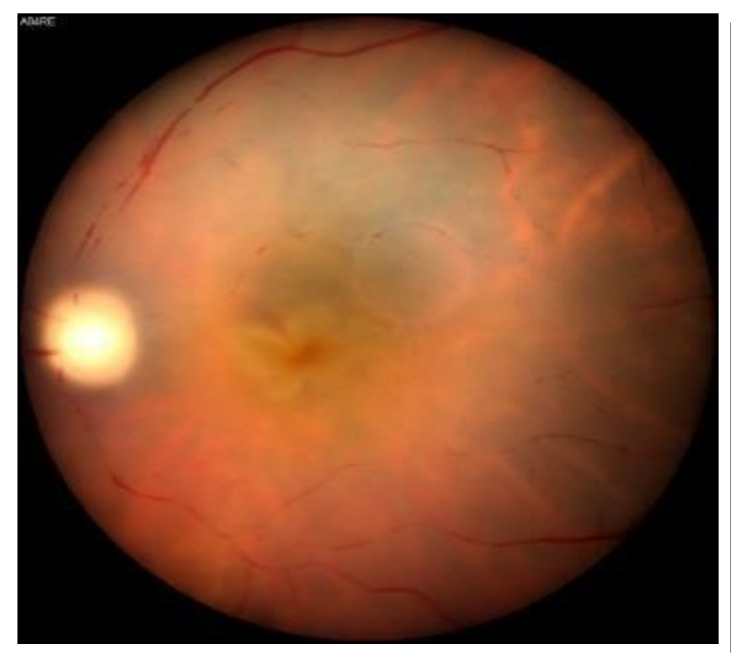

Dissected Choroid Original

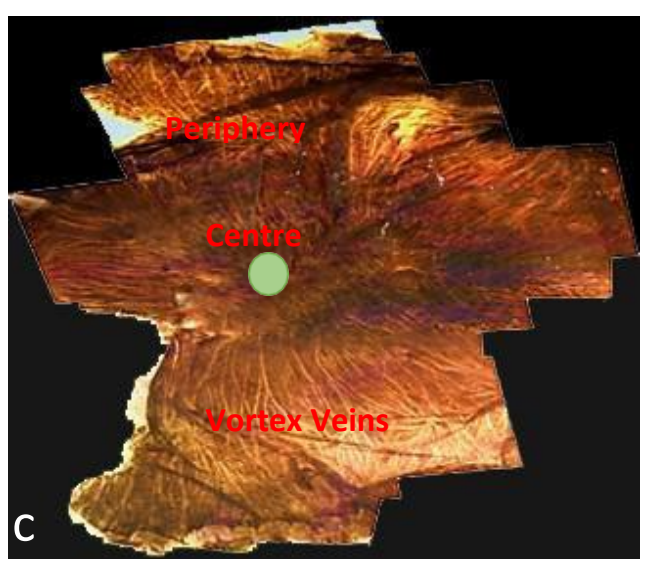

Dissected Retinal Image

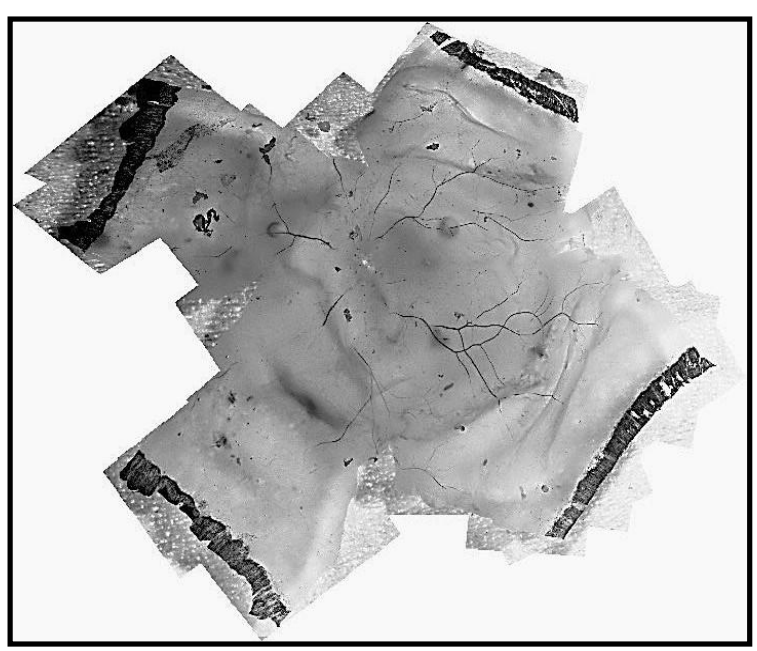

Enhanced Image

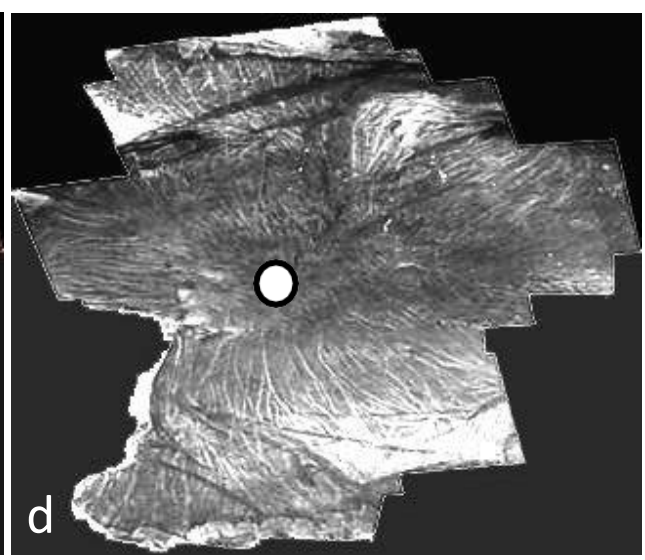


Convolved Image - blood vessels

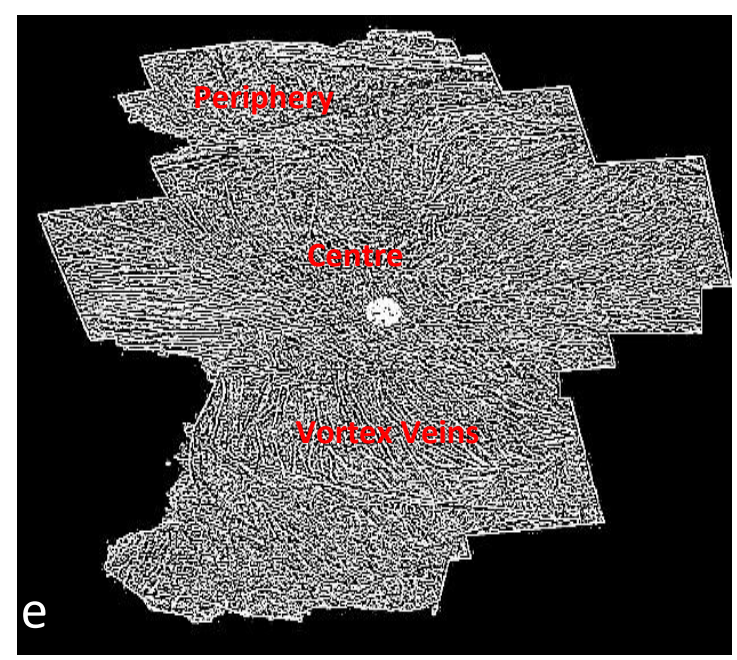

Quantification

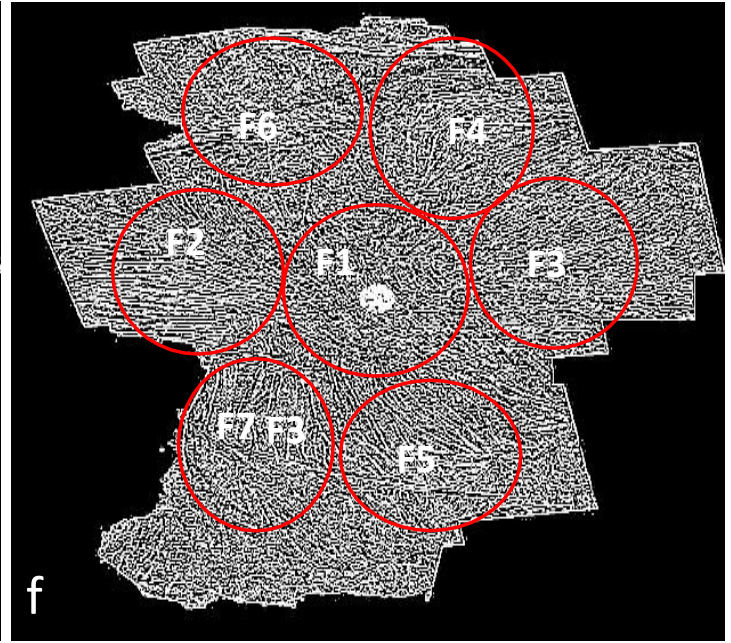

Figure 1-1 Application of convolution algorithm on Normal choroid tissue.

(A)Illustrates fundus image. (B) Isolated Retina layer. (C) Isolated choroid layer and (D) grey scale converted choroid image. (E) Choroid image after application of the convolution algorithm and $(F)$ is the quantification of blood vessels in different areas in the choroid.

\section{Central retina Peripheral retina}

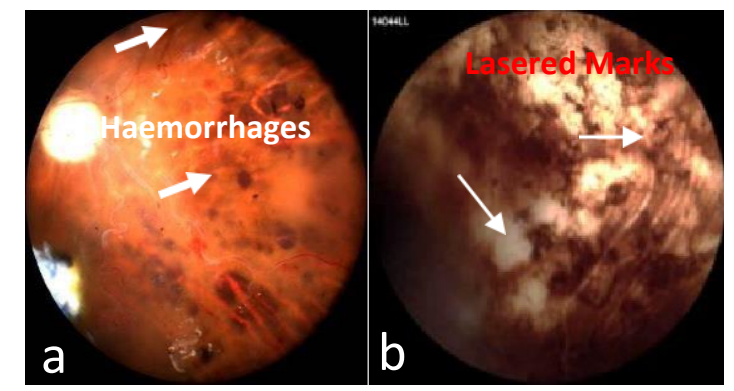

Dissected Choroid

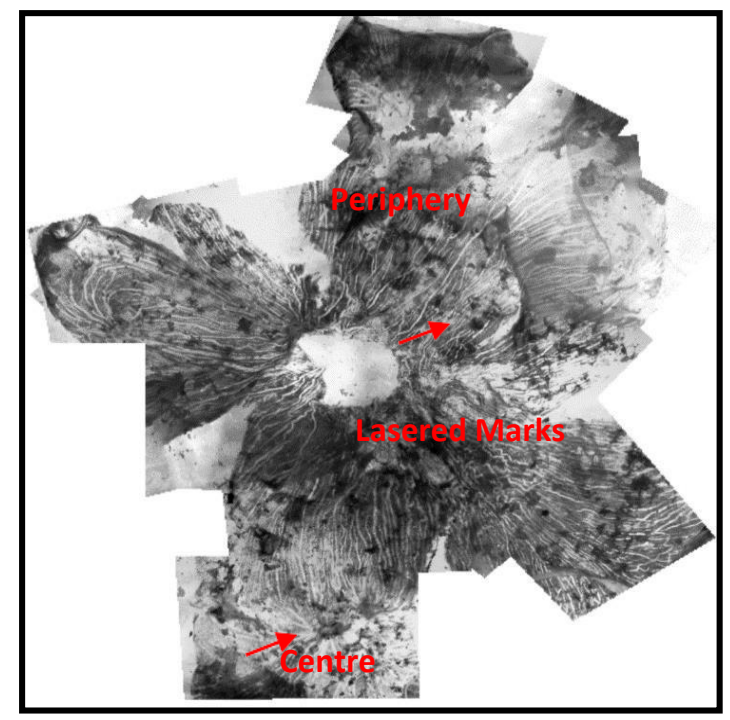

Figure 1-2 Quantification of blood vessels in DR donor tissues using the convolution algorithm

(A, B) Central and peripheral fundus images of a DR donor retina. (C) Isolated choroidal layer in grey scale showing laser treatment marks. (D) After application of the algorithm DR choroid grey scale image showing laser marks as dark spaces. 


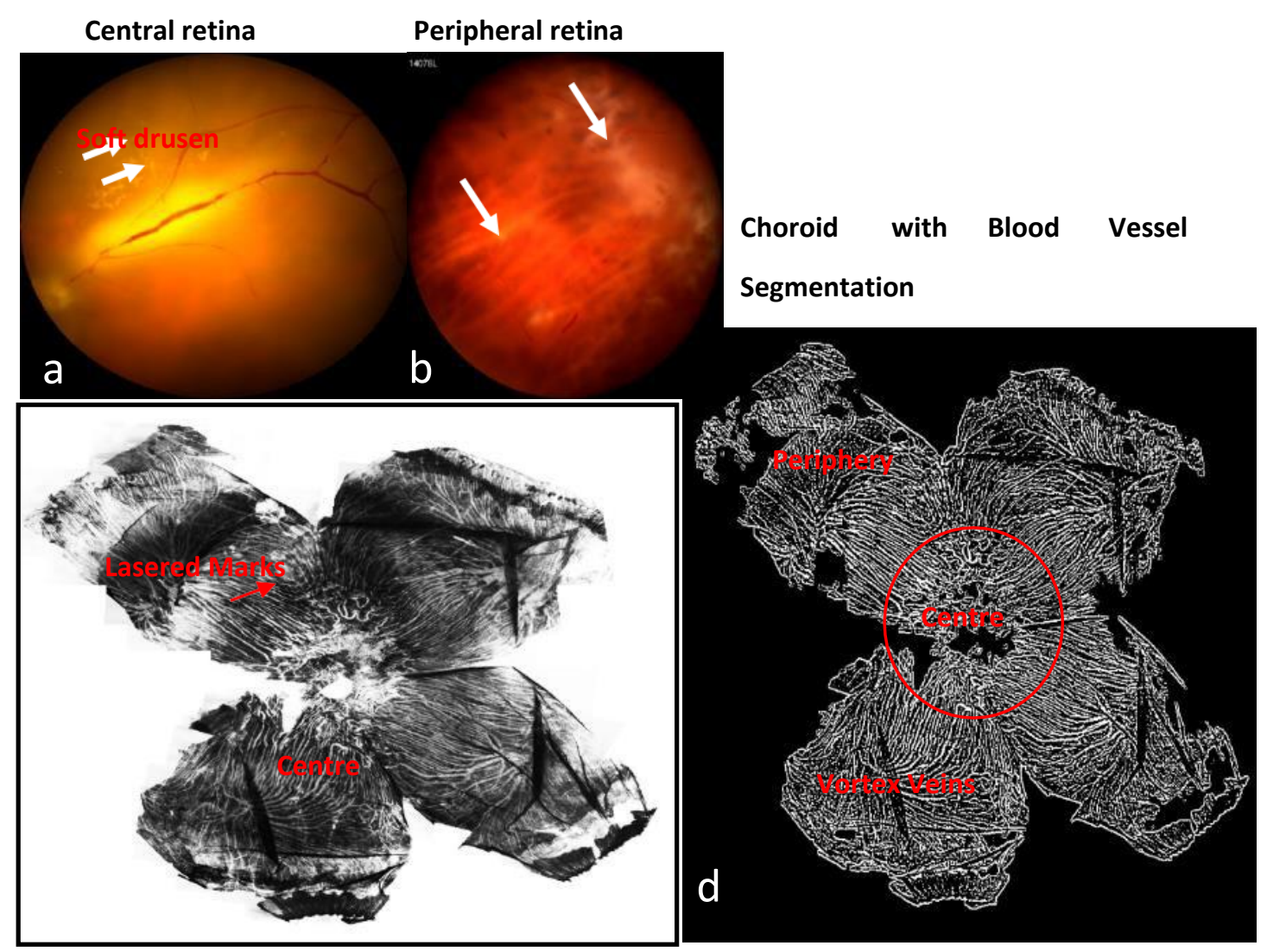

Dissected Choroid

Figure 1-3 Quantification of blood vessels in AMD donor tissues using the convolution algorithm

(A, B) Fundus images of central and peripheral retina illustrating signs of $A M D$, soft drusens and laser marks (represented by arrows on A and B images). (C) Grey scale illustration of AMD choroid. (D) Convolution algorithm application shows the blood vessel structure changes in the AMD choroid.

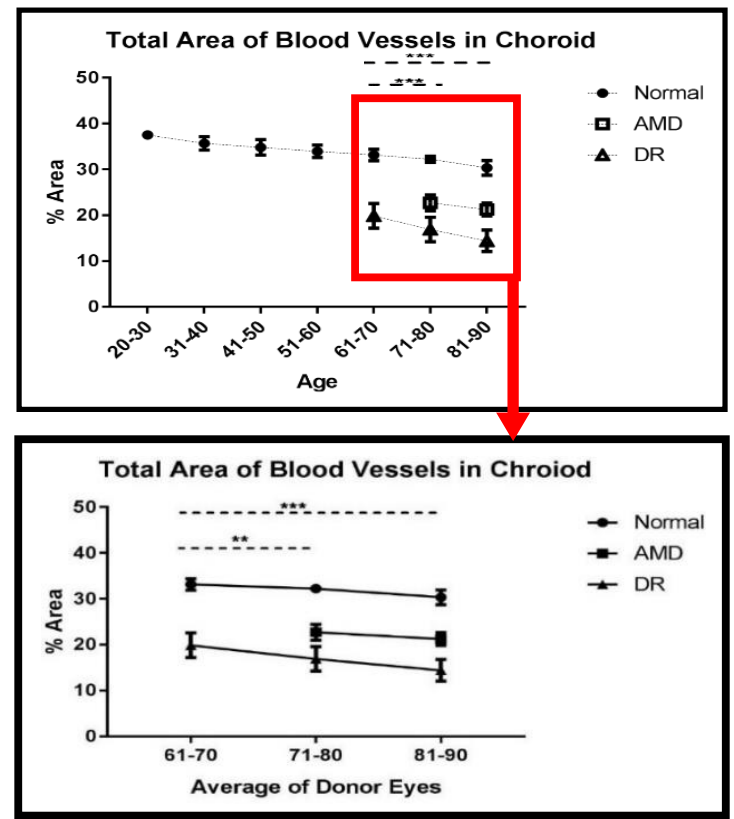

Figure 1-4 Total area of blood vessel quantification in normal and diseased choroid 
Statistical analysis was performed using one-way ANOVA followed by a Bonferroni post hoc test.

Statistically significance was noted in comparison of the blood vessel density in the normal choroids with age * $p<0.01 ;{ }^{* *} p<0.001$ and ${ }^{* * *} p<0.0001$.

Statistically significance was also noticed between normal and diseased age matched choroids blood vessel density. (Normal Vs AMD $p<0.0001$ ) and (Normal Vs DR $p<0.0001$ ).
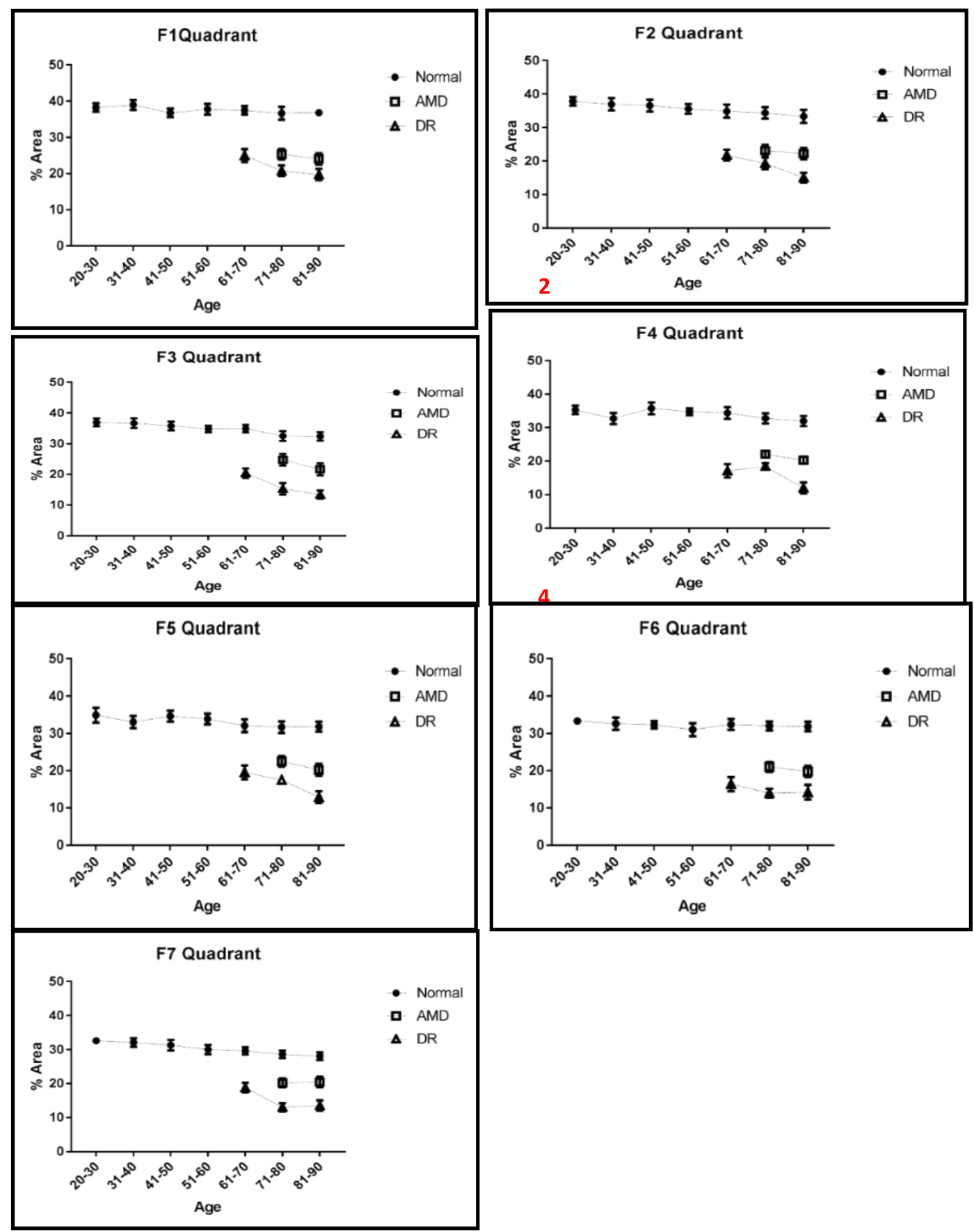

Figure 1-5 Choroidal blood vessel density changes across F1-F7 quadrants in normal and diseased tissues Statistical analysis was performed using one-way ANOVA followed by a Bonferroni post hoc test. Statistical significance was noted in comparison of the normal and diseased choroids (Normal Vs AMD $p<0.0001$ ) and (Normal Vs DR $p<0.0001)$ 

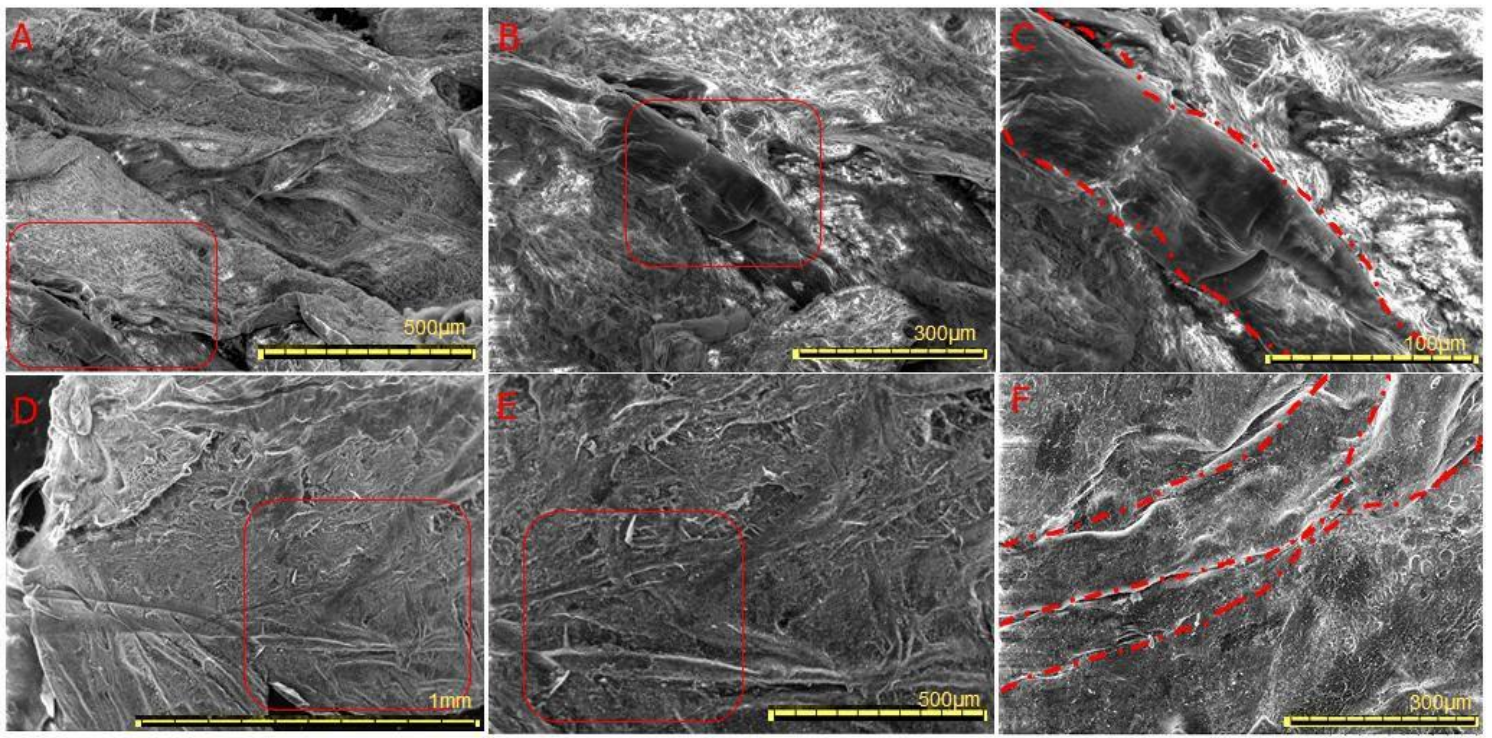

Normal Donor

16-024/Male/

Caucasian/25yrs

Figure 1-6 SEM imaging of the normal donor tissue

(A-C) Morphological illustration of the normal 25-year-old male choroid tissue (D-F), representation of the normal 80year-old female donor tissue. (A-F) red boxed areas in each case showing successive enlargements of the images
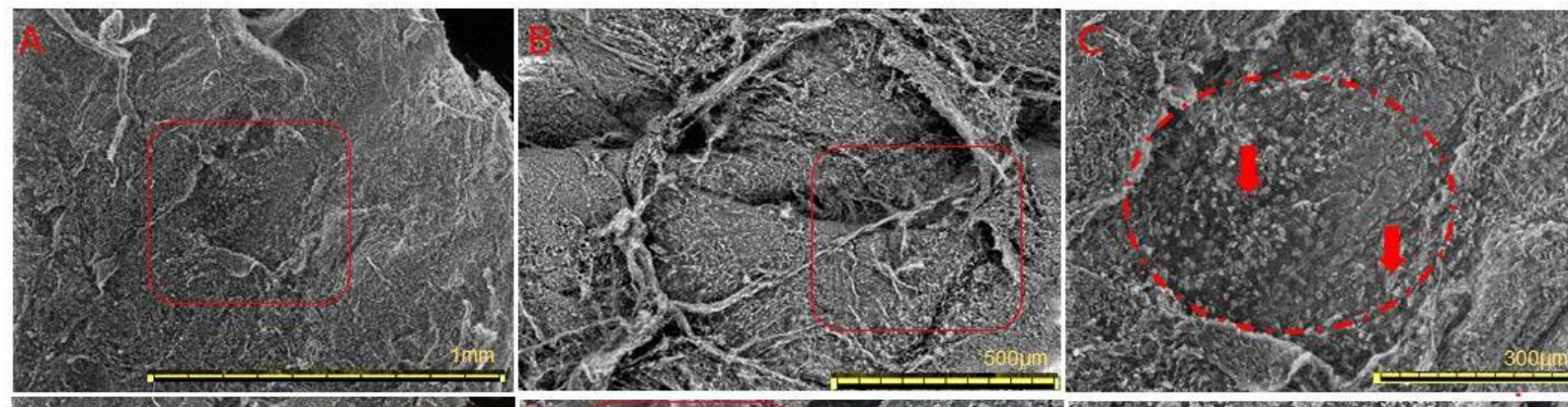

AMD Donor

Normal Donor

15-166/Female/

Caucasian/80yrs
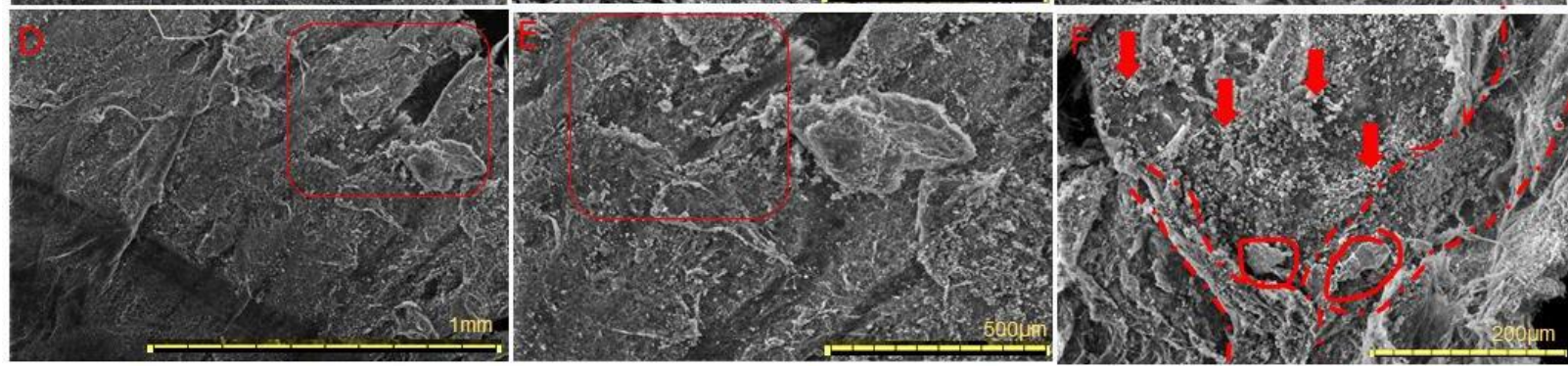

15-04/Male/

Caucasian/

$73 y r s$

AMD Donor

16-013/

Female/

Caucasian/

$83 y r s$

Figure 1-7 SEM imaging of AMD donor tissue showing drusen

(A-C) images of choroid tissue is from a 73-year-old male AMD donor and (D-F) is of an 83-year female with the red squares indicating enlarged regions in subsequent images. Images ( $C$ and $F$ ) shows drusen indicated by the red arrows. In image $F$, dotted red lines represents the larger blood vessels. 


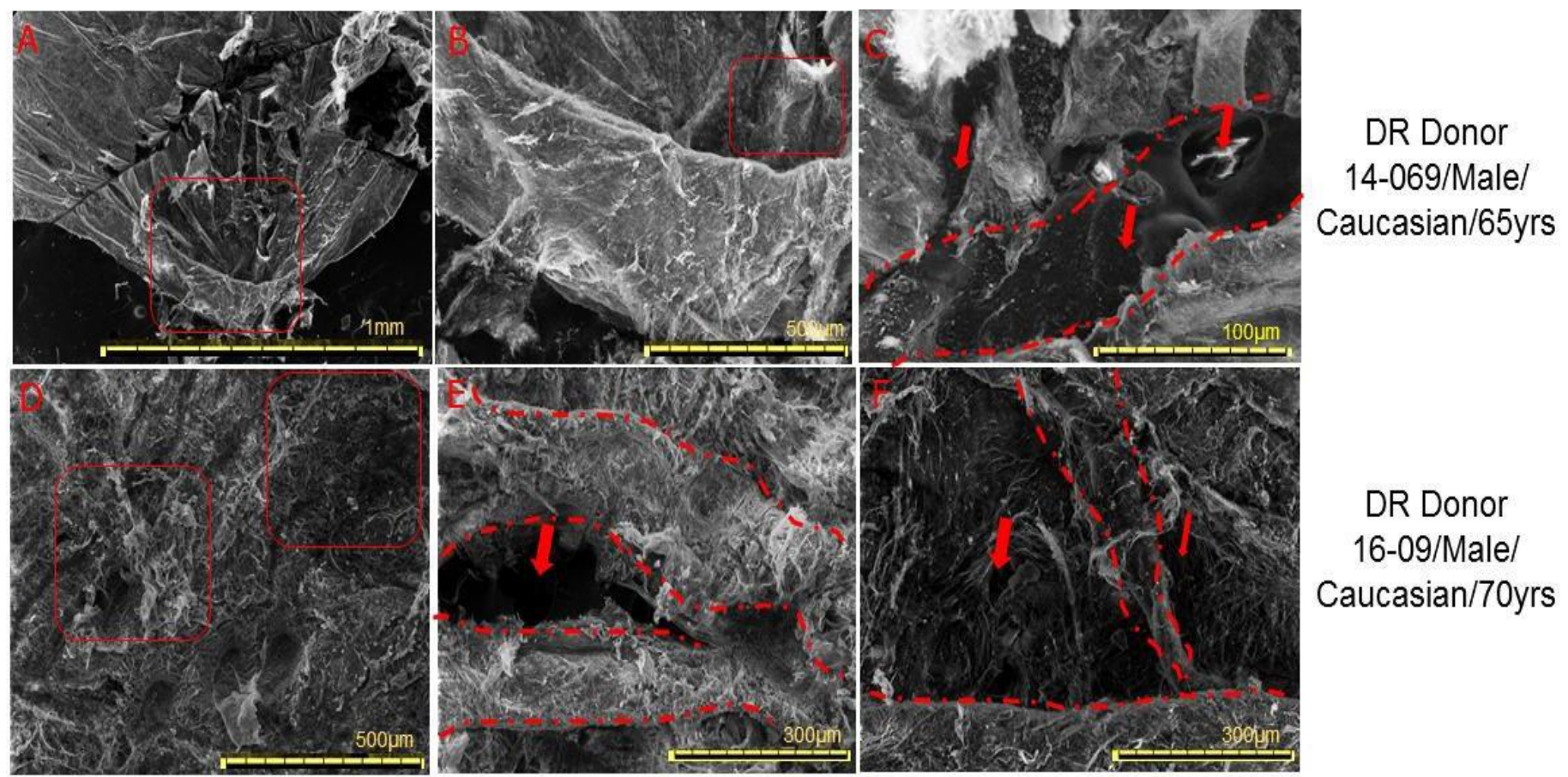

Figure 1-8 SEM imaging of DR donor tissue showing laser treated marks in choroid

(A-C) images are from a 65-year-old male DR tissues and (D-F) 70-year-old female choroidal DR tissue (B) laser marks appear as deep spaces and holes affecting the structure of the matrix and blood vessel walls (example illustrated in image (C) by a dotted red line). (D-F) enlarged images show disorganised matrix and holes within the DR laser treated choroids. (Examples of the blood vessel disorganisation are marked as red dotted lines in the images $\mathrm{C}, \mathrm{E}$ and $\mathrm{F}$ )

\section{DISCUSSION}

Literature shows availability of various imaging modalities to understand and read the retinal tissue. These imaging modalities include Optical Coherence tomography, which uses shorter wavelength of light for better resolution of images. Microscopic techniques are essentially useful in the identification of the structural changes occur in the retina. The SEM is a microscopic imaging technique useful in understanding the underlying cellular and subcellular changes occurred in the retinal and choroidal tissues. The imaging modalities are useful in grading the different stages of the retinal pathologies such as in AMD and DR. ${ }^{36,37}$

Studies have shown that AMD donor eyes have alternations in the Retinal Pigment Epithelium, Bruch's membrane and choriocapillaris in the early stages of the disease. ${ }^{38}$ This study shows, in normal donor tissues there were no difference in the choroid tissue composition but in the AMD tissues, small drusens, confluent deposits underneath RPE layer and the dense compact tissue absence was noted in the diseased donor choroids. We could visualize the vascular bed distribution disturbances along the drusen changes in these tissues.

Our study supports the theory that in DR donor eyes, the composition of the complete choroid was disturbed post-laser treatment. We have noted significant blood vessel loss, holes in between the matrix, disorganised vascular bed pattern, especially underneath the lasered areas. Previous study done in DR donor eyes shows same results as our study with matrix and different cellular structure loss. ${ }^{39}$

This study shows convolution is a simple mathematical algorithm helps in the identification of blood vessels. The computerised algorithm is useful in quantifying the choroidal blood vessel bed. Literature evidence show usage of the blood vessel segmentation technique onto retina tissues. These computerised mathematical models are used to evaluate the progression of the diseases AMD and DR. ${ }^{40}$ These models also help in retinal blood vessel identification, blood vessel edge detection and to extract their borders. Most 
recent studies have shown usage of the segmentation methods in clinics; especially in imaging modalities such as OCT angiography(41). This study successfully used the mathematical algorithm technique to detect and identify blood vessels of the choroid vascular structures through segmentation model. For screening and diagnosing retinal diseases such as AMD and DR. The time and computational power of the kernel increased the accuracy of the study.

It is, unfortunately, not possible to compare this quantitative approach with other studies, as these have not applied a comparable approach for the choroid. The nearest equivalents are the studies of 41, 42 who used OCT angiography instead of a segmentation method such as used here, or studies such as ${ }^{43}$ who used a stereomicroscopic examination of APase incubated choroids using an image analysis program coupled with histology. Both studies, however, revealed a significant reduction in the choroidal vascular bed with disease, consistent with the results reported in this study.

The limitations of the study include true Positive and false positive values of the images in comparison with other algorithms or data sets. It is also important to notice the sensitivity and specificity with other observers which assess the agreement of the individual pixel classification with presence of vessel and non-presence of vessel.

\section{REFERENCE}

1. Kanski JJ, Bowling B. Clinical ophthalmology: a systematic approach: Elsevier Health Sciences; 2011.

2. Girmens J-F, Sahel J-A, Marazova K. Dry age-related macular degeneration: A currently unmet clinical need. Intractable \& Rare Diseases Research. 2012;1(3):103-14.

3. Grunwald J HS, DuPont J. Effect of aging on foveolar choroidal circulation. Arch Ophthalmol. 1998;116:150-4.

4. AYoung RW. Pathophysiology of agerelated macular degeneration. Survey of ophthalmology. 1987;31(5):291-306;
5. BMartinez GS, Campbell AJ, Reinken J, Allan $B C$. Prevalence of ocular disease in a population study of subjects 65 years old and older. Am J Ophthalmol. 1982;94(2):181-9. Epub 1982/08/01.

6. Saleh MD, Eswaran C, Mueen A. An automated blood vessel segmentation algorithm using histogram equalization and automatic threshold selection. Journal of digital imaging 2011;24(4):564-72.

7. ALee SJ, McCarty CA, Taylor HR, Keeffe JE. Costs of mobile screening for diabetic retinopathy: a practical framework for rural populations. Australian Journal of Rural Health. 2001;9(4):18692;

8. BTaylor HR, Keeffe JE. World blindness: a 21st century perspective. British Journal of Ophthalmology. 2001;85(3):261-6;

9. CStreeter L, Cree MJ. Microaneurysm detection in colour fundus images. Image Vision Comput New Zealand. 2003:280-4

10. ASussman EJ, Tsiaras WG, Soper KA. Diagnosis of diabetic eye disease. Jama. 1982;247(23):3231-4;

11. BSiva Sundhara Raja D, Vasuki S. Automatic Detection of Blood Vessels in Retinal Images for Diabetic Retinopathy Diagnosis. Computational and Mathematical Methods in Medicine. 2015;2015:11.

12. ASoares JV, Leandro JJ, Cesar RM, Jelinek HF, Cree MJ. Retinal vessel segmentation using the 2-D Gabor wavelet and supervised classification. IEEE Transactions on medical Imaging 2006;25(9):1214-22;

13. BJelinek HF, Cree MJ, Leandro JJ, Soares JV, Cesar RM, Luckie A. Automated segmentation of retinal blood vessels and identification of proliferative diabetic retinopathy. JOSA A. 2007;24(5):1448-56.

14. ACree $M$, Cornforth $D$, Jelinek $H$. Vessel segmentation and tracking using a twodimensional model. IVC New Zealand. 2005:345-50; 
15. BCree MJ, Leandro J, Soares J, Cesar Jr R, Tang $G$, Jelinek $H$, et al. Comparison of various methods to delineate blood vessels in retinal images. 2005;

16. CCarnimeo L, Bevilacqua V, Cariello L, Mastronardi G, editors. Retinal vessel extraction by a combined neural network-wavelet enhancement method. International Conference on Intelligent Computing; 2009: Springer;

17. DMartins $\mathrm{ClO}$, Medeiros $\mathrm{F}$, Veras RM, Bezerra FN, Cesar R, editors. Evaluation of retinal vessel segmentation methods for microaneurysms detection. Image Processing (ICIP), 2009 16th IEEE International Conference on; 2009: IEEE.

18. Bek T, Jensen PK. Three-dimensional structure of human retinal vessels studied by vascular casting. Acta Ophthalmologica. 1993;71(4):506-13.

19. AMarín D, Aquino A, Gegúndez-Arias $M E$, Bravo JM. A new supervised method for blood vessel segmentation in retinal images by using gray-level and moment invariants-based features. IEEE Transactions on medical imaging. 2011;30(1):146-58;

20. BDua S, Kandiraju N, Thompson HW, editors. Design and implementation of a unique blood- vessel detection algorithm towards early diagnosis of diabetic retinopathy. Information Technology: Coding and Computing, 2005 ITCC 2005 International Conference on; 2005: IEEE;

21. CFraz MM, Remagnino $P$, Hoppe $A$, Uyyanonvara B, Rudnicka AR, Owen CG, et al. Blood vessel segmentation methodologies in retinal images-a survey. Computer methods and programs in biomedicine. 2012;108(1):407-33.

22. Salem NM, Nandi AK. Novel and adaptive contribution of the red channel in pre-processing of colour fundus images. Journal of the Franklin Institute. 2007;344(3):243-56.23. Baecker V. Image processing and analysis with ImageJ and
MRI Cell Image Analyzer. Montpellier RIO Imaging. 2008.

24. ANekovei R, Sun Y. Back-propagation network and its configuration for blood vessel detection in angiograms. IEEE Transactions on Neural Networks. 1995;6(1):64-72;

25. BHoover A, Kouznetsova V, Goldbaum M. Locating blood vessels in retinal images by piecewise threshold probing of a matched filter response. IEEE Transactions on Medical imaging. 2000;19(3):203-10;

26. CPizer $S M$, Johnston RE, Ericksen JP, Yankaskas BC, Muller KE, editors. Contrast-limited adaptive histogram equalization: speed and effectiveness. Visualization in Biomedical Computing, 1990, Proceedings of the First Conference on; 1990: IEEE;

27. DNiemeijer M, Staal J, van Ginneken B, Loog $M$, Abramoff MD, editors. Comparative study of retinal vessel segmentation methods on a new publicly available database. SPIE medical imaging; 2004: SPIE.

28. AHossain F, Alsharif MR, editors. Image enhancement based on logarithmic transform coefficient and adaptive histogram equalization. Convergence Information Technology, 2007 International Conference on; 2007: IEEE;

29. BHossain MF, Alsharif MR, Yamashita K, editors. Medical image enhancement based on nonlinear technique and logarithmic transform coefficient histogram matching. Complex Medical Engineering (CME), 2010 IEEE/ICME International Conference on; 2010: IEEE.

30. AWalter T, Klein J-C, editors. Segmentation of color fundus images of the human retina: Detection of the optic disc and the vascular tree using morphological techniques. International Symposium on Medical Data Analysis; 2001: Springer; 
31. BSopharak A, Uyyanonvara B, Barman S, Williamson TH. Automatic detection of diabetic retinopathy exudates from non-dilated retinal images using mathematical morphology methods. Computerized medical imaging and graphics. 2008;32(8):720-7;

32. CAquino A, Gegúndez-Arias ME, Marín D. Detecting the optic disc boundary in digital fundus images using morphological, edge detection, and feature extraction techniques. IEEE transactions on medical imaging. 2010;29(11):1860-9.

33. Akram MU, Khan SA. Multilayered thresholding-based blood vessel segmentation for screening of diabetic retinopathy. Engineering with Computers. 2013;29(2):165-73.

34. ABerry E. A Practical Approach to Medical Image Processing. Medical Physics and Biomedical Engineering. Leeds,UK: Taylor \& Francis Group; 2013;

35. BAbood ZM, Yousif IA, Hussein AK. Find a Text in Image File Using Correlation Method.

36. ALitts KM, Messinger JD, Freund KB, Zhang $Y$, Curcio CA. Inner Segment Remodeling and Mitochondrial Translocation in Cone Photoreceptors in Age-Related Macular Degeneration With Outer Retinal Tubulation. Investigative Ophthalmology \& Visual Science. 2015;56(4):2243-53;

37. BKlaassen I, Van Noorden CJF, Schlingemann RO. Molecular basis of the inner blood-retinal barrier and its breakdown in diabetic macular edema and other pathological conditions. Progress in retinal and eye research. 2013;34:19-48. 38. Biesemeier A, Taubitz T, Julien S, Yoeruek E, Schraermeyer U. Choriocapillaris breakdown precedes retinal degeneration in age-related macular degeneration. Neurobiology of Aging. 2014;35(11):2562-73.

39. Toussaint DD, Dustin PP. Electron microscopy of normal and diabetic retinal capillaries. Archives of Ophthalmology. 1963;70(1):96-108.

40. Shreyasi Hazra AP, Tuhin Utsab Paul. A Comparative Study of Retinal Image Processing Technique for Blood Vessel Segmentation. International Journal of Science and Research (IJSR). 2013;5(4). Epub April 2016.

41. Liu C, \& Wang, R. K. . Automated choroidal segmentation method in human eye with 1050nm optical coherence tomography. . In Progress in Biomedical Optics and Imaging - Proceedings of SPIE 2014;8942.

42. Zhang L, Lee $K$, Niemeijer M, Mullins RF, Sonka M, Abràmoff MD (2012) Automated Segmentation of the Choroid from Clinical SD-OCT. Investigative Ophthalmology \& Visual Science 53:7510-7519.

43. McLeod DS, Taomoto M, Otsuji T, Green WR, Sunness JS, Lutty GA (2002) Quantifying changes in RPE and choroidal vasculature in eyes with age-related macular degeneration. InvestOphthalmol Vis Sci 43:1986-1993. ${ }^{6}$

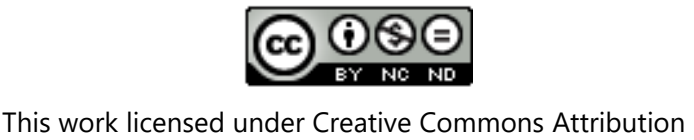

